\title{
Characteristics of Younger and Older Adults with Hospital-Acquired Delirium: a Claims Data Study Spanning 14 years
}

J Gen Intern Med 36(4):1150-2

DOI: $10.1007 / \mathrm{s} 11606-020-06379-9$

(C) Society of General Internal Medicine 2021

\section{INTRODUCTION}

Delirium is an acute change in mental status with a fluctuating course, and has been associated with high morbidity and mortality. ${ }^{1}$ While delirium may affect patients of any age, most delirium prevention initiatives in the hospital focus on older adults as they are more vulnerable to developing delirium. ${ }^{2}$ Little is known about delirium in younger adults. In this study, we compare the characteristics of younger (18-64 years) and older ( $\geq 65$ years) adults with hospital-acquired delirium using claims data from 2003 to 2017.

\section{METHODS}

Data for this study were obtained from Optum Clinformatics ${ }^{\mathrm{TM}}$ Datamart. This database comprises administrative health claims from a national managed care company affiliated with Optum and Medicare Advantage Part D members. It includes 15-18 million annual covered lives and contains de-identified clinical information abstracted from medical records, pharmacy, and medical claims.

We included 14,775,245 adult patients who were discharged from a hospital between December 31, 2003, and December 31, 2017. Delirium was identified using International Classification of Disease (ICD) codes. We excluded 497,720 patients who had a diagnosis of delirium present on admission. Our Institutional Review Board exempted this study from review.

Bivariate analysis was conducted to assess differences in the characteristics between younger (18-64 years) and older $(\geq$ 65 years) adults with delirium. For each characteristic, standardized mean difference (SMD) for each variable was calculated using the method implemented in the "tableone" package in R. SMD is defined as the mean difference between the two groups over the pooled standard deviation. SMD of 0 implies no difference was observed between the two groups for that

Received April 21, 2020

Accepted December 1, 2020

Published online January 8, 2021 variable. The higher the SMD, the greater the magnitude of difference between the variables. Analysis was done using R, version 3.6.1.

\section{RESULTS}

A total of 3,233,358 patients with hospital-acquired delirium were identified. Of these, $27.5 \%$ were younger adults (Table 1). The rate of delirium was $10.8 \%(890,957$ of $8,230,662)$ in younger adults and $35.8 \%(2,342,401$ of $6,544,583)$ in older adults $(\mathrm{SMD}=0.61)$. Compared to older adults with delirium, younger adults with delirium received more opiates (18.6\% versus $10.0 \%)$ and antipsychotics $(4.2 \%$ versus $1.1 \%)$, had lower mean Charlson comorbidity score (1.5 versus 2.0$)$, and lower rates of heart failure (13.6\% versus $28.3 \%$ ), renal disease $(11.5 \%$ versus $20.9 \%)$, and dementia $(0.8 \%$ versus $8.3 \%)$.

Compared to older adults with delirium, younger adults with delirium were more often discharged home $(86.5 \%$ versus $67.0 \%$ ) and less often discharged to a rehabilitation facility (5.5\% versus $23.7 \%)$. Thirty-day mortality was lower among younger adults $(4.2 \%$ versus $8.3 \%)$ but no significant difference in the length of stay or hospitalization cost was noted between younger and older adults with delirium.

\section{DISCUSSION}

To our knowledge, this is the largest study assessing the differences between younger and older adults who were diagnosed with delirium during their hospital stay. Our study shows high rates of hospital-acquired delirium in younger $(10.8 \%)$ and older adults (35.8\%). Approximately 1 in 4 cases of delirium occurred in a younger adult (18-64 years old). LOS and cost were similar in younger and older adults with delirium.

This study has a few limitations. First, the population is restricted to those in the Optum Clinformatics ${ }^{\mathrm{TM}}$ Datamart comprised of commercial health plan and Medicare Advantage members, thus excluding uninsured younger adults and those with an alternate insurance (e.g., Medicaid) and older adults without Medicare part D coverage. However, since this is a large database with over 65 million covered lives from a geographically diverse area, the results are likely generalizable. Second, we used ICD codes for identifying patients with delirium. Bedside assessment for delirium would yield more 
Table 1 Characteristics of Patients with Delirium

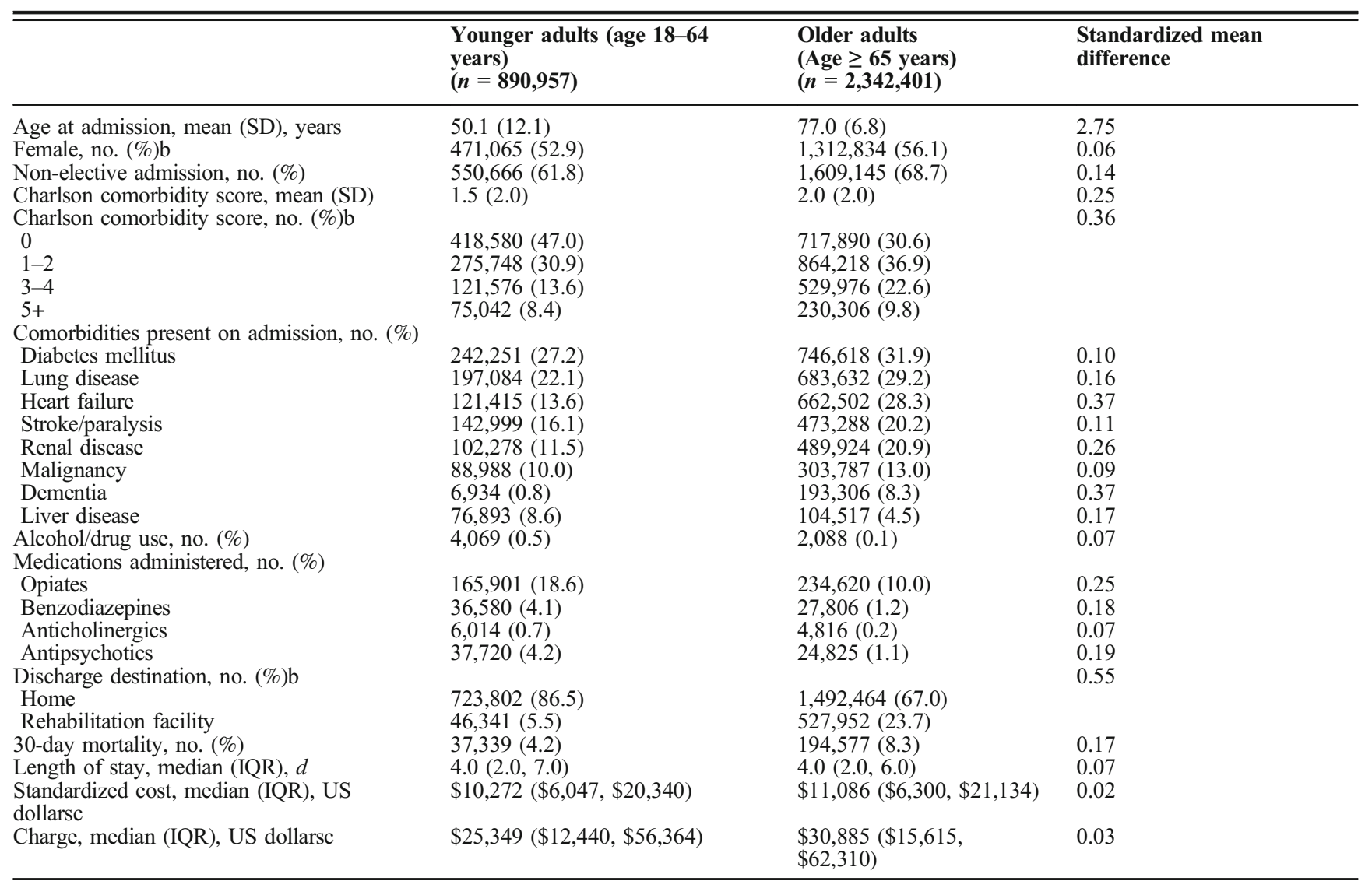

$S D$, standard deviation; IQR, interquartile range

${ }^{a}$ The following International Classification of Disease (ICD) codes were used to identify patients with delirium: ICD-9-Clinical modification codes included 293.0, 780.79, 780.1, 780.97, 307.9, 348.3, 348.30, 348.39, 349.82, 348.31, 298.9, 294.11, 294.21, 294.9, 298, 297, 293.1, 780.02, 292.81, 290.41, 799.23, 799.51, 293.82, 293.81, 293.89, 293.9, 290.3, 290.20, 290.11, 290.12. The ICD-10 codes included F05, F05.0, F05.1, F05.8, F05.9, R41.0, F06.0, F06.2, F06.8, F09, F11.121, F11.122, F11.150, F11.151, F11.159, F11.221, F11.222, F11.129, F11.229, F11.23, F11.250, F11.251, F11.259, F11.920, F11.921, F11.922, F11.950, F11.951, F11.959, F11.929, F11.93, F13.239, F13.230, F13.121, F13.150, F13.151, F13.159, F13.231, F13.232, F13.250, F13.251, F13.259, F13.921, F13.931, F13.932, F13.950, F13.951, F13.959, F13.939, F13.930, F13.929, F13.920, F13.129, F13.120, F29, F22, F23, F24, R40.0, R41.0, R41.82, R41.89, R41.9, G93.4, G93.40, G93.49, G93.41, R40.4, R44, R44.0, R44.1, R44.2, R44.3, R44.8, R44.9, R45.1, R45.6, R45.87, R41.840

${ }^{b}$ Missing values for sex: 151 in the 18-64 years group and 244 in the $\geq 65$ years group; missing values for Charlson comorbidity score: 11 in the 18 64 years group and 11 in the $\geq 65$ years group; missing values for discharge destination: 53,965 in the 18-64 years group and 115,570 in the $\geq 65$ years group

${ }^{c}$ Definitions used in the Optum Clinformatics database: Standardized cost was defined as an estimate of the allowed amount for the facility charges related to that admission. Charge was defined as the amount the facility requested to be reimbursed for the service(s) provided

accurate diagnoses but is not possible in large claims databased studies. Third, the data on alcohol and drug use is likely under-documented but we surmise that affected both groups equally. Fourth, we could only report the variables included in the database.

There is paucity of literature on delirium in younger adults. Few small studies that reported delirium in younger adults were restricted to oncology or palliative care patients and posthematopoietic stem cell transplant recipients. ${ }^{3-5}$ In our study, delirium among patients 18-64 years of age was relatively common (10.8\%) and was associated with similar LOS and cost as in older adults with delirium. Delirium prevention initiatives in the hospital should include high-risk younger adults along with older adults.
Nidhi Rohatgi, $M D, M^{1,2}$

Yingjie Weng, $M H S^{3,2}$

Neera Ahuja, $M D^{1,2}$

Maarten G. Lansberg, $\mathrm{MD}, \mathrm{Ph} \mathrm{D}^{4,2}$

${ }^{1}$ Division of Hospital Medicine, Department of Medicine, Stanford University School of Medicine,

Stanford, CA, USA

${ }^{2}$ Stanford, CA, USA

${ }^{3}$ Quantitative Sciences Unit, Division of Biomedical Informatics Research, Department of Medicine, Stanford University School of Medicine,

Stanford, CA, USA

${ }^{4}$ Department of Neurology and Neurological Sciences, Stanford University School of Medicine,

Stanford, CA, USA 
Corresponding Author: Nidhi Rohatgi, MD, MS; Stanford, CA, USA (e-mail: nrohatgi@stanford.edu).

\section{Compliance with Ethical Standards:}

Conflict of Interest: The authors declare that they have no conflict of interest.

\section{REFERENCES}

1. Siddiqi N, House AO, Holmes JD. Occurrence and outcome of delirium in medical in-patients: a systematic literature review. Age Ageing. 2006;35(4):350-364. https://doi.org/10.1093/ageing/afl005

2. Inouye SK, Bogardus ST, Baker DI, Leo-Summers L, Cooney LM. The Hospital Elder Life Program: a model of care to prevent cognitive and functional decline in older hospitalized patients. Hospital Elder Life
Program. J Am Geriatr Soc. 2000;48(12):1697-1706. https://doi.org/10. $1111 /$ j. 1532-5415.2000.tb03885.x

3. Elsayem AF, Bruera E, Valentine AD, et al. Delirium frequency among advanced cancer patients presenting to an emergency department: A prospective, randomized, observational study. Cancer. 2016;122(18):29182924. https://doi.org/10.1002/cncr.30133

4. Fann JR, Hubbard RA, Alfano CM, Roth-Roemer S, Katon WJ, Syrjala KL. Pre- and post-transplantation risk factors for delirium onset and severity in patients undergoing hematopoietic stem-cell transplantation. J Clin Oncol. 2011;29(7):895-901. https://doi.org/10.1200/JCO.2010.28. 4521

5. Weckmann MT, Bay C, Abu Ata N, Morrison RS. Incidence and Cause of Delirium in Hospitalized Patients between the Ages of 18 and 56: A Retrospective Chart Review. Int J Palliat Care. 2014;2014:1-7. https://doi. org/10.1155/2014/214265

Publisher's Note: Springer Nature remains neutral with regard to jurisdictional claims in published maps and institutional affiliations. 